\title{
The use of brain-computer interfacing for ambient intelligence
}

\author{
Gangadhar Garipelli ${ }^{12 \star}$, Ferran Galán ${ }^{13}$, Ricardo Chavarriaga ${ }^{1}$ Pierre W. \\ Ferrez $^{12}$, Eileen Lew ${ }^{12}$, and José del R. Millán ${ }^{12}$ \\ 1 IDIAP Research Institute, Martigny, Switzerland. \\ 2 Ecole Polytechnique Fédérale de Lausanne, Lausanne, Switzerland. \\ 3 University of Barcelona, Barcelona, Spain.
}

\begin{abstract}
This paper is aimed to introduce IDIAP Brain Computer Interface (IBCI) research that successfully applied Ambience Intelligence $(\mathrm{AmI})$ principles in designing intelligent brain-machine interactions. We proceed through IBCI applications describing how machines can decode and react to the human mental commands, cognitive and emotive states. We show how effective human-machine interaction for brain computer interfacing (BCI) can be achieved through, 1) asynchronous and spontaneous BCI, 2) shared control between the human and machine, 3) online learning and 4) the use of cognitive state recognition. Identifying common principles in BCI research and ambiance intelligence (AmI) research, we discuss IBCI applications. With the current studies on recognition of human cognitive states, we argue for the possibility of designing empathic environments or devices that have a better human like understanding directly from brain signals.
\end{abstract}

\section{Motivation}

Brain Computer Interfacing (BCI) or Brain Machine Interfacing (BMI) refers to interaction with devices, where user's intentions represented as several brain states are deciphered and translated into actions without requiring any physical action [44] [25] [21]. There is a growing interest in the use of brain signals for communicating and operating devices, which is facilitated by the advances in the the measurement technologies in the past decades. As BCI bypasses the classical neuromuscular communication channels, this technology is intended to use for rehabilitation of tetraplegic or paraplegic patients to improve their communication, mobility and independence. The BCI research also opens up new possibilities in natural interaction for able-bodied people (e.g., for space applications, where environment is inherently hostile and dangerous for astronauts, who could greatly benefit from direct mental teleoperation of external semi-automatic manipulators [26], and for entertainment applications like multimedia gaming [20]). Typical applications of BCI are communication aids such as spelling devices [5] [31] [25] and mobility aids such as wheelchair [41]. In the current paper, we

\footnotetext{
* Gangadhar.Garipelli@idiap.ch
} 
introduce the design of IDIAP BCI (IBCI) towards intelligent interaction and empathic devices and show how its key features are consistant with Ambient Intelligence (AmI) design principles.

The vision of AmI is designing digital environments in which the electronics devices are sensitive to people's needs, personalized to their requirements, anticipatory of their behavior and responsive to their presence. The main goals of AmI based interaction are, 1) understanding human function and sensory information, 2) analysis of sensory information, 3) design of human-like-reasoning devices and intelligent interaction. The BCI research brings a new sensing modality for tracking neurophysiological information related human's cognitive and emotive sates. The brain computer interaction can be seen also as a new mode of interaction similar to speech and vision based interaction, but with mental commands directly from the brain signals. So the general principles of interaction for destining AmI are also valid for the design of interaction with BCI.

Since we are interested in designing interactions between intelligent systems (human user and intelligent machine), it is natural to derive ideas from humanhuman communication. These ideas offer a starting point for a quest for new forms of interaction. According to Schmidt [39], for interaction, the context information is important. In particular, the key components are,

- Shared knowledge: In communication between intelligent systems, a common knowledge is essential for understanding each other. This common knowledge is extensive and is usually not explicitly mentioned. In most cases, this common knowledge includes world or environment model.

- Communication error recovery: Communication between intelligent systems may not be error free, many conversations include short term misunderstandings and ambiguities. But the misunderstandings are often detected by monitoring the response of the communication partner. In case of misinterpretation the dialogs are repeated and corrected.

- Surrounding situation and context: Communication and interaction between intelligent systems will happen in a specific situation. Inclusion of contextual information (e.g., a model of environment) provides common ground with implicit conventions.

In the current paper, we review IBCI applications that incorporated the principles for interaction. In particular, the interaction of IBCI-system is designed with the following components, 1) shared knowledge between the robot and user. The ongoing work on recognition of human anticipatory behavior described in section 4 is based on this principle. For example, consider a scenario of an intelligent robotic wheelchair facing a dining table in a hall of several tables. From the robot-controller's point of view, the table is an obstacle and it can't decide by it self whether to dock to it or to avoid it. But it is the user who decides to dock to it if he wants to take brakefast. The user anticipates for the docking event to happen if he wishes to dock. The shared knowledge allows the robot to make corresponding actions (e.g., docking, or avoiding the obstacle) upon the recognition of anticipation related brain activity of the user. The shared knowledge, i.e., robot's detection of a table and user's anticipation of events allows to achieve the 
desired goal. 2) communication error recovery through feedback and the detection of error related brain activity. We have implemented these two mechanisms in our applications that allow the user to correct his commands from the feedback of recognized commands by classifiers ( described in 3) as well as the robot to change its commands up on the recognition of error related brain activity (described in 4) and 3) context filtering of illogical mental commands inferred by the interface. For a brain actuated robot application (described in section 3.1), the filtering is achieved by using a finate state machine that translated the mental commands into device commands according to the environmental context. In the case of a brain actuated wheelchair application (described in section 3.2), the filtering is achieved by combining the probabilities inferred by the classifier for mental commands with that of context-based-filter of the robotic wheel chair.

In the next section we review the state of art of BCI research along with the methods that lead to the success of IBCI. In section 3, we review IBCI applications that implement the key principles introduced above. In section 4, we show the possibility of designing empathic devices with the recognition of user's cognitive states directly from brain signals. Finally in section 5 we discuss conclusions and future directions of research.

\section{BCI research and IBCI system}

A schematic of a BCI system is shown in Figure 1. Brain electrical activity is acquired using electrodes (either implanted inside the brain or externally on the scalp). From the recorded signals, features (e.g., amplitudes of evoked potentials, or sensory motor cortex rhythms) that reflect user's intent, are extracted using signal processing methods. These features are then translated into device commands (e.g., using neural networks) which are then issued to systems like, virtual-keyboards, mobile robots, robotic wheelchairs and computer games. Feedback from these systems is given to the user using various modalities (e.g., visual, auditory etc.).

$\mathrm{BCI}$ is broadly classified into three categories based on invasiveness of the recording technique as 1) invasive, 2) partially invasive and 3) non-invasive BCI [22]. For an invasive BCI, the electrodes are implanted directly into the grey matter of the brain during neurosurgery. As they rest in the grey matter, it can produce the highest quality signals of BCI devices but are prone to scar-tissue build-up, causing the signal to become weaker or even lost as the body reacts to a foreign object in the brain [19]. Partially invasive BCI [10] uses electrodes implanted inside the skull but resting outside the brain rather than amidst the grey matter (e.g., Electrocorticography, ECoG). They produce better resolution than non-invasive electrodes and have lower risk of forming scar-tissue in the brain than fully invasive electrodes. Finally, Electroencephalograph (EEG), Magnetoencephalography (MEG) and functional magnetic resonance imaging (fMRI) have both been used successfully in non-invasive BCI. Among all the EEG is the most used signal acquisition method mainly due to its fine temporal resolution, ease of use, portability and low set-up cost. Since the current paper is based on 


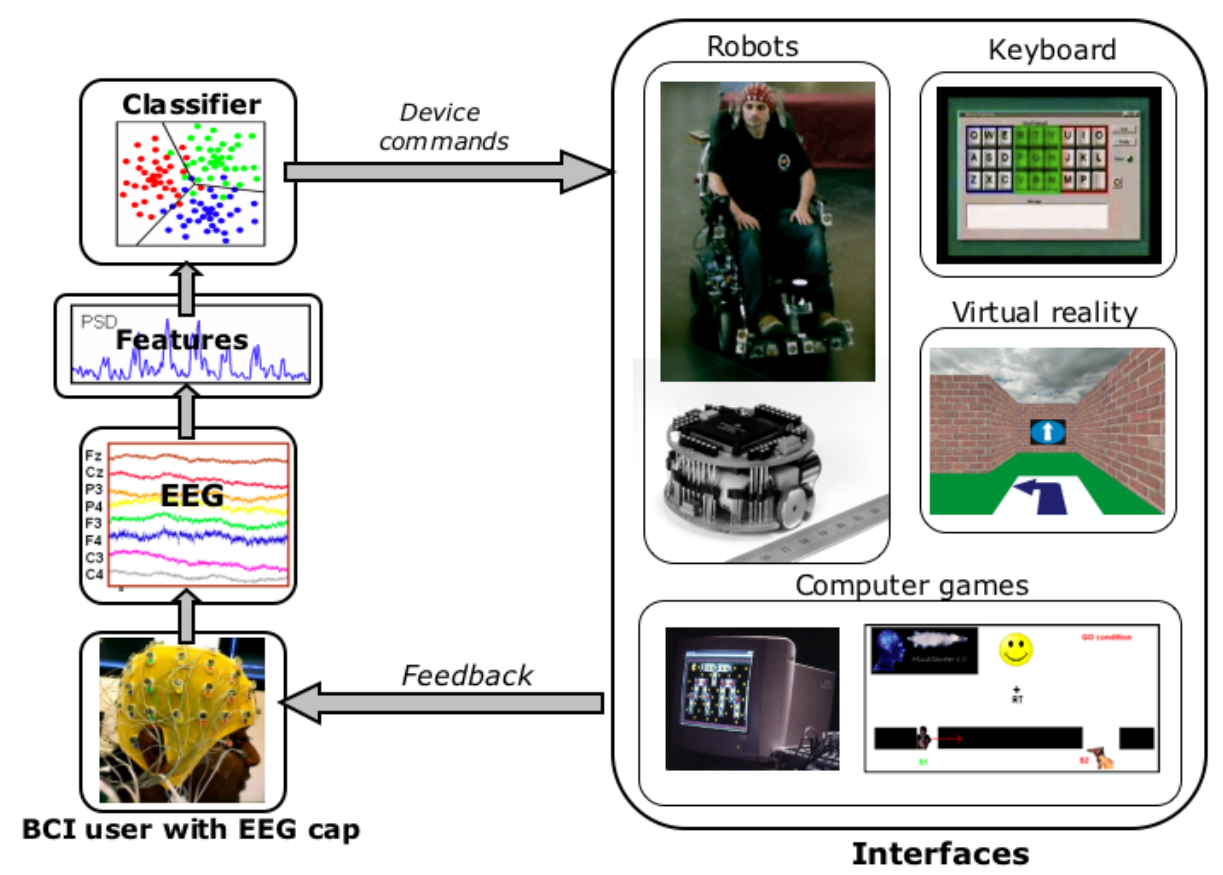

Fig. 1. Operation of a brain computer interfacing (BCI) system.

non-invasive IBCI system, we review feature extraction and classification stages of EEG based BCI.

Based on the operation, non-invasive BCI systems can be classified into two types, 1) system driven, 2) user driven. The system driven BCI uses EEG waveforms that are generated automatically in response to external stimulus (e.g., visual, auditory stimulus from the interfacing machine), called evoked potentials (EP). One example is the P300 signal, which is a potential evoked by an awaited infrequent event that appears at centro-parietal locations along the mid line of the scalp. It is a positive wave peaking around $300 \mathrm{~ms}$ after task-relevant stimuli [36]. Traditionally, P300 has been used in BCI research to develop virtual keyboards [2] [9] with a typing speed of five letters per minute, but recently this same potential has also been the basis for brain-actuated control of a virtual reality system [4] and of a wheelchair [36]. Steady-state visual evoked potentials (SSVEP) are another example of evoked potentials that are induced by a visual stimulus repeated at a rate higher than $6 \mathrm{~Hz}$ [40]. Most SSVEP-based BCI system depend on muscular control of gaze direction for their operation, whereas all other kinds of BCI systems do not depend on the brain's normal output pathways of peripheral nerves and muscles [15] [24] [40]. The main drawback of system driven BCI is that, since the subject's response is locked to the stimulus, he cannot generate mental commands at any time he wants. 
On contrast the user-driven BCI is based on self modulation of EEG rhythms by the user, i.e. spontaneous brain activity. for instance, self modulation by imagination of movements can result in changes in EEG rhythm in central region of the scalp overlying the sensorimotor cortex [3] [8] [33] [45]. These rhythms are the basis of several BCI systems [3] [8] in which imagination of hand movement gives rise to an amplitude suppression in the $\alpha$-band $(8-12 \mathrm{~Hz})$ and $\beta$-band $(13-28 \mathrm{~Hz})^{4}$ rhythms over the contralateral primary hand motor cortical area [33]. Wolpaw and co-workers [43] [45] used continuous changes in the amplitudes of these rhythms to move a cursor in a computer screen. Alternatively, some researchers measure slow cortical potentials (SCP) whose negative amplitudes are related to the overall preparatory excitation level of a given cortical network, the more negative the more active over the top of the scalp at electrode- $\mathrm{Cz}$ [5] [18]. Attentional modulation seems to constitute the cognitive strategy in the physiological regulation of SCP. The team lead by Birbaumer has widely shown that healthy subjects as well as severely paralyzed patients can learn to self-control their SCPs through operant conditioning to move an object on a computer screen in a BCI referred to as Thought Translation Device (TTD) [17].

EEG-based BCIs are limited by a low channel capacity. Most of the current systems have a channel capacity below 0.5 bits/s [43]. One of the main reasons for such a low bandwidth is that they are based on synchronous protocols, where EEG is time-locked to externally paced cues repeated every 4-10 s and the response of the BCI is the average decision over this period (system driven BCI) [5] [31] [34] [37] [43]. The system dribven BCI is not natural for the user since his response is always time-locked to externally placed cues generated by the system. The user can't not decide by him self whenever he want to make a decision. On the contrary, the IBCI group utilizes more flexible asynchronous protocols where the subject makes self-paced decisions (user-driven) on when to stop performing a mental task and start immediately the next one [27] [30] [29]. In such asynchronous protocols, the subject can voluntarily change the mental task (e.g., imagination hand movement. See figure 2(b) for scalp topographies of EEG activity during these mental tasks in $\alpha$ band) being executed at any moment without waiting for external cues (this approach is grounded in a number of neurocognitive studies that have found that different mental tasks such as mental rotation of geometric figures [46], arithmetic operations [7], or language [32] activate local cortical areas to a different extent). The time of response of an asynchronous IBCI can be below 1 second (responds every 0.5 second) [30]. The rapid responses of asynchronous BCIs, together with their performance, give a theoretical channel capacity between 1 and $1.5 \mathrm{bits} / \mathrm{s}$.

Coming to the feature extraction, IBCI team analyzes continuous variations of EEG rhythms over several frequency bands. The user specific EEG-features

\footnotetext{
${ }^{4}$ EEG activity is typically described in terms of rhythmic activity. The rhythmic activity is divided into several frequency bands (e.g., $\alpha$ band from 8 to $12 \mathrm{~Hz}$. Suppression in this band power is usually observed in sensory motor areas while the user performing mental imagination.)
} 


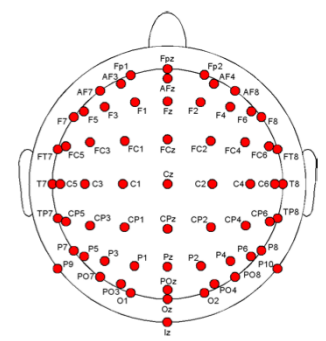

(a)

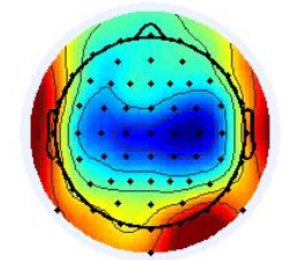

Imagination of left hand

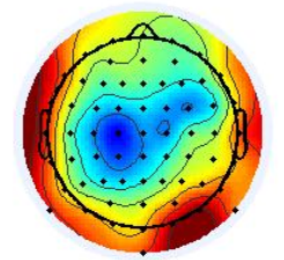

Imagination of right hand

(b)

Fig. 2. (a) Top view of electrode positions according to the international 10-20 system. (c) Event related de-synchronization: decreased $\alpha$-band $(12 \mathrm{~Hz})$ power contralaterally to the imagination of hand. Dark regions correspond to lower power.

extracted using Canonical Variate Analysis (CVA) for multi-class problems [13]. This technique maximizes the separability between the patterns generated by executing the different mental tasks. For the classification of these features, IBCI team typically uses Gaussian classifiers which are a modified Gaussian Mixture Model (GMM) [28] [29]. The output of the classifier is posterior probability that can be used to label specific classes and an 'unknown' class. It is worth noting that the use of statistical rejection criteria helps to deal with an important aspect of a BCI, namely idle states where the user is not involved in any particular mental task. These classifiers has been shown to perform better than support vector machines (SVMs), temporal processing neural networks (NNs) [16], committees of multi later perceptrons (MLPs), learning vector quantization and incremental radial basis networks [28] (for more details on the Gaussian classifier, refer [28], for a review of classification engines for BCI in general, refer Lotte et al [23]).

To sum up, the key principles behind the feature extraction and classification parts of IBCI system are, 1) using task induced EEG rhythms over cortical areas as features of mental commands, 2) canonical feature extraction for multi-class problems, 3) statistical classifier with rejection criteria and 4) asynchronous and spontaneous operation. Current research of IBCI group also focuses on adding 'cognitive states recognition' to 'mental command recognition' to improve its performance. The recognition of cognitive states can be used for implementing the principles of intelligent interaction like, shared knowledge and error recovery as described in section 1 . The details of recognition of the cognitive states are described in section 4. In the next section, we discuss the design of brain actuated interaction to drive a mobile robot and a robotic wheelchair in natural environments.

\section{IBCI applications}

In this section we present the current applications of IBCI system for controlling a mobile robot and a robotic wheelchair through intelligent interaction in the 


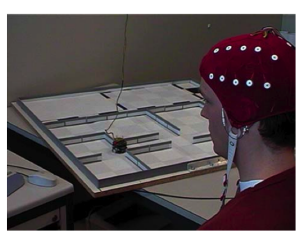

(a)

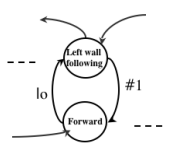

(b)

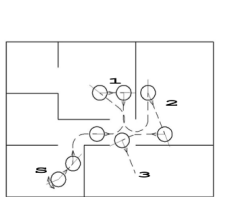

(c)

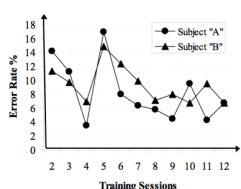

(d)

Fig. 3. Brain actuated robot control: (a) a user driving brain actuated robot (b) a fragment of Finate State Automaton (FSA) used for the shared control. Transitions between the 6 behaviors were determined by 3 mental states (\#1: turn left, \#2: turn right, \#3: go forward), 6 perceptual states (lo: leftwall, ol:right wall, ö: wall or obstacle in front), and some memory variables.) (c) Experimental platform and a typical trajectory followed by the robot under the mental control. The robot started in bottom left room and then visited three other rooms, (d) error rate (percentage of false positives)(adopted from [30]).

light of AmI with the following key components, 1) shared control, 2) error control through detection of error related potentials, 3) inclusion of contextual information.

\subsection{Brain-actuated robots}

Recently, IBCI group has shown for the first time that asynchronous analysis of EEG signals is sufficient for humans to continuously control a mobile robot (i.e. Khepera) along non-trivial trajectories requiring fast and frequent switches between mental tasks [30]. Human users learned to mentally drive the robot between rooms in a house-like environment (see Figure 3(a)). Furthermore, mental control was only marginally worse than manual control on the same task. A key element of this brain-actuated robot is shared control between two intelligent agents (i.e., the human user and the robot). The user only gives high-level mental commands (e.g., turn left, turn right, forward) that the robot performs autonomously. Another critical feature is that a BCI asynchronous operation, allowing the user to issue mental commands at any moment.

In order to endow the system with flexible, robust control, there is no oneto-one mapping of user's mental commands and robot's actions. Instead, we combine environmental information gathered from robot's on-board sensors with mental commands to take appropriate actions according to the context (i.e. shared control). This combination is implemented by a Finite State Automation (FSA) [30]. The transitions between different behaviors are determined by the 3 mental commands, 6 perceptual states of the environment (based on the robots sensory readings: left wall, right wall, wall or obstacle in front, left obstacle, right obstacle, and free space) and a few internal memory variables. These perceptual states are determined by using a neural network classifier that takes input from the sensory readings [30]. The memory variables keep contextual information required to implement correctly the different behaviors. Figure 3(b) shows a 
Table 1. Time in seconds for three different trials in controlling first mentally and then manually by two users.

\begin{tabular}{|c|c|c||c|c|}
\hline \multirow{2}{*}{ Trial } & \multicolumn{2}{|c|}{ User-A } & \multicolumn{2}{c|}{ User-B } \\
\cline { 2 - 5 } & Mental-control & manual-control & Mental-control & Manual-control \\
\hline \hline 1 & 149 & 124 & 219 & 156 \\
2 & 183 & 135 & 189 & 155 \\
3 & 191 & 129 & 175 & 117 \\
\hline Average & 174 & 129 & 194 & 143 \\
\hline
\end{tabular}

Table 2. Comparison of bit-rate of online classification with static initial classifier.

\begin{tabular}{|c|c|c|}
\hline Session \# & Static initial classifier & Online classification \\
\hline \hline 1 & 0.29 & 1.44 \\
2 & 0.20 & 1.41 \\
3 & 0.14 & 1.34 \\
4 & 0.18 & 1.34 \\
\hline \hline Average & $0.20 \pm .06$ & $1.38 \pm 0.05$ \\
\hline
\end{tabular}

fragment of the FSA (for full description, see [30]). As shown in the figure, if the robot is performing the behavior forward and perceives a wall to the left, it switches automatically to the behavior follow left wall. The transition to the behavior-forward is necessary, for example, in the case where the robot is approaching an open door and the user wants the robot not to enter into the room.

A final element is the use of an appropriate feedback indicating the current mental state recognized by the embedded classifier. This is done by means of three lights (red, blue, green) on top of the robot that corresponds to the three mental commands (turn right, turn left, move forward). Thus, if the robot is following the left wall and is approaching an open door, a blue feedback indicates that the robot will turn left to continue following the left wall (and, so, it will enter into the room). On the contrary, a green feedback indicates that the robot will move forward along the corridor when facing the doorway and will not enter into the room. This simple feedback allows users to correct the robot trajectory in case of errors in the recognition of the mental states or errors in the execution of the desired behavior (due to the limitations of the robot sensors). The figure 3(c) shows a typical trajectory of brain actuated robot. After 5 and 3 days of initial training with the interface, the users achieved a satisfactory level of performance (correct recognition was above $65 \%$ while the errors were below 7\% - the remaining were 'unknown' response) (see Figure 3(d)). Table 3.1 gives the time in seconds necessary to generate the desired trajectories in three different trials for the two participants comparing mental control and mannual control. Remarkably, trial duration for mental control was comparable with mannual control. On average, brain-actuated control of the robot takes only 
$35 \%$ longer than manual control for both the participants. The figure $3(\mathrm{~d})$ shows the performance curve two users. First, a clear improvement can be observed during the first day (sessions 2 to 4), with an excellent performance. Second, the performance degrades at the beginning of second day (session 5) but recovers at the end. This shows difficulty of generalizing from one day to the next due to natural variability of brain signals. This variability can be compensated by incorporating online-learning as discussed in following paragraphs.

The variability of EEG signal within a session and from session to session is due to several factors including the background activity, fatigue and concentration levels, and intentional change of subject's strategy. This means that the classifier designed with past data might not perform well for the present or future data. To deal with this problem, IBCI applies adaptive algorithms that are constantly tuned to the subject. These techniques improve the performance of the BCI system allowing the subject to learn to use BCI more effectively. We first build classifier with the past data and then, as new EEG is obtained during the use of the BCI, we use it for updating the classifier (for more details, refer [1] [6]).

The studies on online learning are performed on offline data collected during brain-actuated robot control. The improvements in terms of the bit-rate comparing static initial and adaptive classifier are shown in table 3.1 (bit rate is channel capacity as explained in [29]). The online classification rates are much higher than the static classifiers. Moreover, the classifiers obtained at the end of each session (i.e. that were modified online throughout the experiment) outperforms the initial classifier.

From the above brain-actuated robot application, and consistent with the AmI principles, we conclude that, 1) fusing of knowledge of the human user and intelligent robot allows for effective human-computer interaction; 2) apart from using shared knowledge and contextual information, the error recovery (achieved by using feedback in the present case) is also important for successful control; 3) online adaption of the intelligent system will improve the interaction performance.

\subsection{Brain actuated wheelchair}

The recent studies of the IBCI in collaboration with KV Leuven under the framework of the European project MAIA (http://www.maia-project.org) aim at the development of a brain-actuated wheelchair that can provide mobility directly through mental control. An autonomous controller embedded in the wheelchair could serve help paralyzed patient for navigation. However, the subject might lose the feeling of continuous control with such a controller. The loss of independence is undesirable and therefore, shared control between the user and the controller is more suitable [35]. IBCI's design for such a system has three basic elements [35] [41], 1) adaptive shared controller that fuses the human and wheelchair decisions in a Bayesian way for better steering commands , 2) context information from the model of environment for filtering out unlikely decisions 


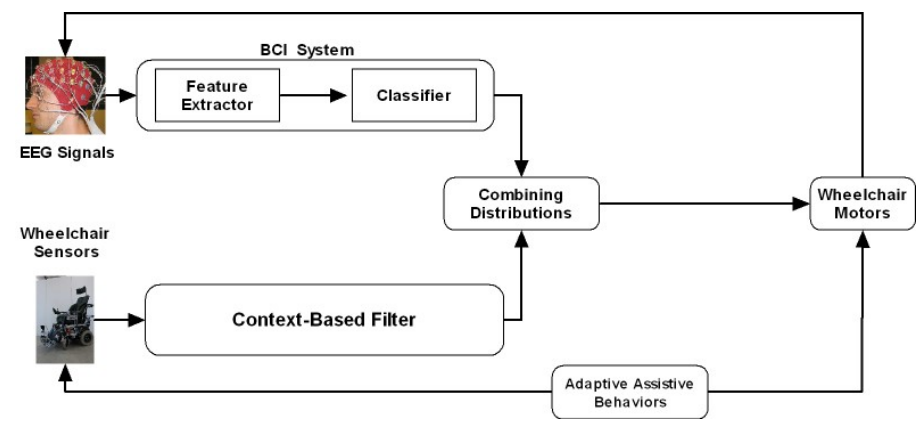

Fig. 4. A schematic of shared control system

taken by the classifier and 3) assistive behaviors (collision avoidance (A0) obstacle avoidance(A1), and orientation recovery (A2)) based on the model of environment (e.g., openings in a corridor). See Figure 4 for the architecture of shared control of brain-actuated wheelchair.

Similar to the brain-actuated robot control, user can steer the wheelchair by issuing three discrete mental commands. The induced EEG rhythms (power spectrum density computed over one second in a selected subset of electrodes and frequency bands [14]) due to these mental commands are classified by a statistical Gaussian classifier whose outputs are posterior probabilities for the device commands ( move forward, turn left and turn right). The asynchronous BCI system responds every 0.5 seconds by sending these probability distribution of the three mental commands to the shared controller which are then translated into steering commands (i.e., translational $(\nu)$ and rotational $(\omega)$ velocity). Instead of directly executing the user's steering commands, the shared control system takes environmental situation into account which is registered through a laser scanner. With this knowledge, the controller triggers one of the assistive behaviors using a winner-takes-all method (e.g., if the user steers too close to an obstacle, an avoidance behavior of the shared control is activated to prevent collision). Studies with the adaptive shared control are illustrated in figure 5(b). Without A2, the subject makes unnecessary loops while navigating (refer to [35]). The average elapsed time and average distance travelled (refer to [35] and [41]) also reduced significantly in navigating towards the goal.

Since the mental command recognition is not perfect we need to correct them using environmental context. This is archived by adding a context filter to adaptive shared controller. The controller estimates the environmental context for detecting illogical steering signals. The context filtering in three steps. First, the context estimation is performed by defining a general, a-priory known model of the user intention (e.g., smooth and efficient forward navigation through the environment) on one hand and a constant automatic estimation of the environmental situation on the other hand. The situations were modelled as the number and location of openings (i.e. wide, open spaces through which user might safely navigate). Second, each opening represents a general direction in which the user 


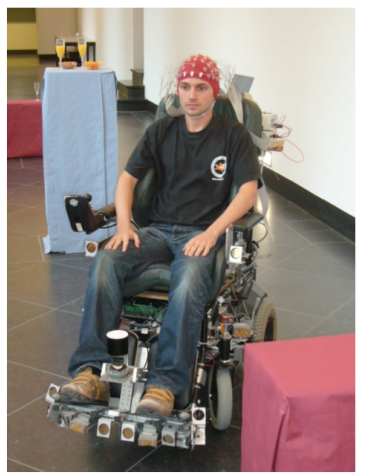

(a)

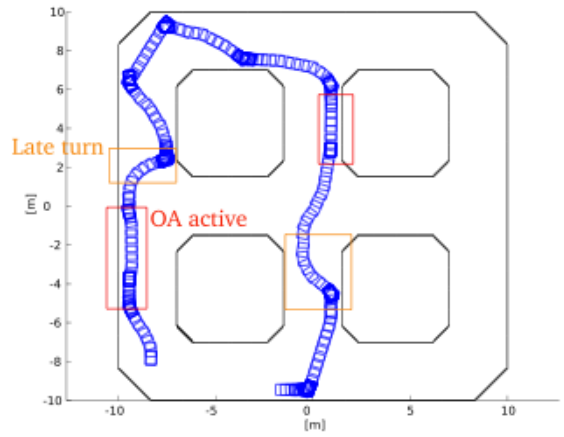

(d)

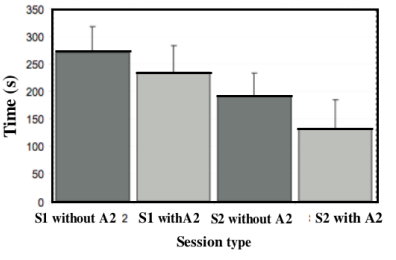

(b)

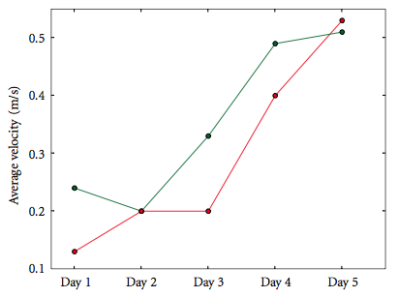

(c)

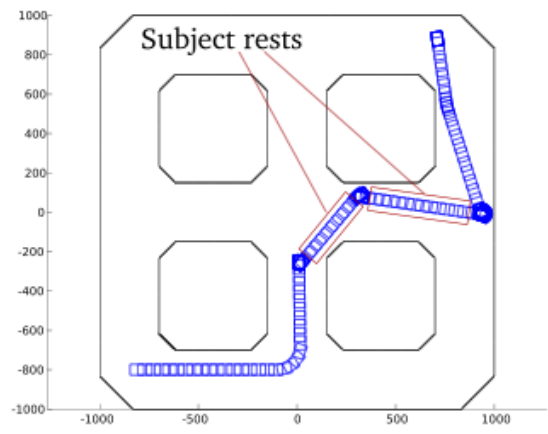

(e)

Fig. 5. (a) The subject wearing an electroencephalogram (EEG) sensor cap maneuvering the robotic wheelchair through a natural indoor environment. The visible are the sensors of the robotic platform: a laser range scanner in front and sonar sensors are visible at the lower part of the wheelchair, (b) Average navigation time with and without orientation recovery (A2) for sessions 1 and 2. (c) Average of velocity during five training days. The lower line represents the performance without context filter and the upper one represents the average velocity when the context filter is active. (d) Wheelchair trajectory in a different environment without context filtering. (e) A trajectory of a session with context filtering (the figure reproduced from [41]).

might opt to continue his travel. With the knowledge of current situation, a probability distribution concerning the possible user actions were built. Third, the intelligent controller combines the probability distributions of the statistical classifier of BCI system and probability distribution generated from the envi- 
ronmental knowledge, so as to get a better estimation of the user's local steering intent.

The performance of the context filtering is also tested in terms of velocity of maneuvering (see figure 5(c)). Inspire of the fact that the user's driving skills improve gradually, both with and without context filtering, activation of this feature allow the user to steer faster in early stages of training. Figure $5(\mathrm{~d})$ shows a trajectory performed without context filtering. We can see that there are many-nearby collisions (collisions won't happen due to A1), resulting in a rather jaggered path. Enabling context filter results in smoother trajectories, although near-collisions still occur (see figure 5(e)). More results on context filtering are described in [41].

\section{Cognitive state recognition: Towards empathic devices}

Cognition is a mental process of knowing, including aspects such as awareness, perception, reasoning, and judgment. An empathic agent is a cognizant that comprehends needs, feelings, problems and views of humans and responds to them. Recently, IBCI group started investigating on the use of brain signals related to cognitive process for boosting the IBCI performance. By implementing recognition of cognitive states, the IBCI becomes a basic empathic agent. In particular the group is investigating on using "user's awareness of machine's error" and "human anticipatory states".

\subsection{Decoding human awareness of machine error}

BCIs are prone to errors in the recognition of user's intent from his mental commands. As in the human-human interaction, an elegant way of improving the BCI performance is to use a verification procedure directly based on presence of error related potentials (ErrP) in the brain activity. ErrP is a potential elicited after presenting the feedback of an error as response which is clearly detected in $\mathrm{FCz}$ and $\mathrm{Cz}$ electrode (see figures 6(a) and 6(b)). Several studies show the presence of this potential in typical choice reaction tasks when the subject makes mistake by himself. At IDIAP, in the context of BCI, we have shown that ErrPs are elicited even when error is made by the interface during the recognition of subject's intension. Ferrez and Millán termed this type of ErrP as interaction$\operatorname{Err} P[11]$ [12], as it is elicited by the presentation of feedback indicating incorrect response of simulated BCI.

Furthermore, we are interested in how ErrP can be used to improve the performance of a BCI. As shown in the Figure 6(c), after translating the subject's intention form his mental command, into control command for the robot, the BCI provides a feedback of it, which will be executed only if no ErrP follows the feedback (see figure 6(c)). In this new interaction method, the challenge is to recognize the ErrP on single trials. After characterization of these potentials, we have developed classification technique that archive successful recognition of these potentials (up to $80 \%$ correct classification of ErrP and up to $83 \%$ of 
correct trials [11]). In addition, this type of interaction improves the bit-rate of the BCI system by $28 \%$ for three-class problem and by $72 \%$ for two-class problem(see [11] for more detailed results).

\subsection{Decoding human anticipation}

Animals have the ability to anticipate to upcoming events given a predictive model. In particular, in humans, the EEG correlates of anticipation are well known, and one of such signal is Contingent Negative Variation (CNV). CNV is an increasing negative shift of the cortical electrical potential associated with anticipated response to an external stimulus. It is therefore interpreted as both an expectation related potential and anticipation related potential [38] [42]. Recognition of CNV can be used for implementing shared knowledge of the human user and a semi-autonomous system in making final decisions. For example, a robotic wheelchair facing a dining-table can not decide by itself whether to dock to it or to avoid it (i.e., obstacle avoidance behavior). But, the presence or absence of anticipation related potentials in the subject's EEG will allow the wheelchair to make a final decision. The question that we are addressing in this section is that, "Is it possible for machines to predict human anticipation to particular events?".

We study changes in the CNV depending on the task-dependent relevance of external stimulus ( $\mathrm{S} 1$ ) in a classical Go/NoGo CNV paradigm. In "Go" condition, the subject is instructed to anticipate to imperative stimulus (S2) and press a key on its arrival and in "NoGo" condition, the subject instructed to do nothing. On-line recognition of such changes provides information that can be used by the semi-autonomous system in situations when it is not able to make reliable decisions. Grand averages of potentials recorded in CNV Go/NoGo paradigm is shown in figure 6(d). These potentials are classified using a simple Linear Discriminant Analysis (LDA) classifier. The results show that the anticipatory potentials can be classified up to an accuracy of $70 \%$ at least $0.5 \mathrm{sec}$ before the subject presses a key.

The recognition of ErrP and anticipatory signals from the EEG introduces empathic capabilities in the BCI system. Thus, we show the feasibility of designing empathic devices that can predict human actions, judgements and needs directly brain signals. Further, the implementation of other emotive states recognition, such as attention, alarms, frustration and confusion will improve empathic capabilities of our BCI system.

\section{Conclusions and future work}

In this paper we have introduced several applications of the IDIAP BCI (IBCI). In particular this paper shows how these applications successfully integrate design principles from human-centered approaches for intelligent interaction in the domain of Brain Computer interfaces. Namely, we have shown how the described IBCI systems are endowed with 1) Shared knowledge, 2) communication error 
recovery and 3) contextual information. These principles are consistant with from AmI design criteria and allows for the robust performance of the IBCI systems by showing strong evidence of the potential synergy between $\mathrm{AmI}$ and BCI research.

Sharing the knowledge between the human user and robot perception of environment is achieved by using FSA for the brain-actuated control of a robot. In the case of brain-actuated wheelchair, it is achieved by combining the probability distributions inferred by the Gaussian classifier from the user's mental commands with those inferred from the environment by the wheelchair sensors. We have shown two possible methods for communication recovery, 1) by giving a feedback of the recognized mental commands to the user so that he can change his mental commands in case of error, and 2) with the use of interaction-ErrP. We have also shown that context filtering of illogical mental commands inferred by the interface improves the driving performance of the wheelchair. The recent work of IBCI team shows a way to improve empathic capabilities of a machine by using human cognitive state recognition (e.g., recognition of ErrPs and anticipation related potentials). These capabilities can be improved by recognizing other cognitive and emotive states such as attentional level, frustration, alarm, and confusion.

In summary, IBCI research shows the feasibility of developing systems that have an enhanced comprehension of human's cognitive and emotive states establishing boosted intelligent human-machine interactions. The synergy between AmI and BCI research will permit to develop empathic systems and environments, providing tools for making human-machine interaction more resemblance to human-human interactions.

\section{Acknowledgements}

This work was supported in part by the Swiss National Science Foundation through the National Centre of Research on "Interactive Multimodal Information Management (IM2)", European IST Programme FET Project FP6-003758, and FP6-IST-027140. This paper only reflects the author's views and founding agencies are not liable for any use that may be made of the information contained herein. We thank 'Division PMA team' of Katholieke Universiteit, Leuven, for it's collaboration on brain-actuated-wheelchair application.

\section{References}

1. Buttfield A., Ferrez P.W., and Millán J. del R. Towards a robust BCI: Error potentials and online learning. IEEE Trans. on Neural Systems and Rehabilitation Engineering, 14(2):164-168, 2006.

2. B. Z. Allison and J. A Pineda. ERPs evoked by different matrix sizes: implications for a brain computer interface (bci) system. IEEE Trans Neural Syst Rehabil Eng, 11(2):110-113, Jun 2003. 
3. F. Babiloni, F. Cincotti, L. Lazzarini, J. del. R Millán, J. Mouriño, M. Varsta, J. Heikkonen, L. Bianchi, and M. G. Marciani. Linear classification of lowresolution EEG patterns produced by imagined hand movements. IEEE Trans Rehabil Eng, 8(2):186-188, Jun 2000.

4. J. D. Bayliss. Use of the evoked potential P300 component for control in a virtual apartment. IEEE Trans Neural Syst Rehabil Eng, 11(2):113-6, June 2003.

5. N. Birbaumer, N. Ghanayim, T. Hinterberger, I. Iversen, B. Kotchoubey, A. Kübler, J. Perelmouter, E. Taub, and H. Flor. A spelling device for the paralysed. Nature, 398(6725):297-8, Mar 251999.

6. Anna Buttfield and José del R. Millán. Online classifier adaptation in braincomputer interfaces. IDIAP-RR 16, IDIAP, 2006.

7. F. Chochon, L. Cohen, P. F. van de Moortele, and S. Dehaene. Differential contributions of the left and right inferior parietal lobules to number processing. $J$ Cogn Neurosci, 11(6):617-630, Nov 1999.

8. G. Dornhege, B. Blankertz, G. Curio, and K. R. Müller. Boosting bit rates in noninvasive eeg single-trial classifications by feature combination and multiclass paradigms. IEEE Trans Biomed Eng, 51(6):993-1002, Jun 2004.

9. L. A. Farwell and E. Donchin. Talking off the top of your head: toward a mental prosthesis utilizing event-related brain potentials. Electroencephalogr Clin Neurophysiol, 70(6):510-523, Dec 1988.

10. E. A. Felton, J. A. Wilson, J. C. Williams, and P. C. Garell. Electrocorticographically controlled brain-computer interfaces using motor and sensory imagery in patients with temporary subdural electrode implants. report of four cases. $J$ Neurosurg, 106(3):495-500, Mar 2007.

11. P.W. Ferrez and J. del R. Millán. You are wrong!-automatic detection of interaction errors from brain waves. In Proceedings of the 19th International Joint Conference on Artificial Intelligence, Edinburgh, UK, August 2005.

12. P.W. Ferrez and J. del R. Millán. Error-related EEG potentials in brain-computer interfaces. In G. Dornhege, J. d. R. Millán, T. Hinterberger, D. McFarland, and K.-R. Müller, editors, Towards Brain-Computer Interfacing. The MIT Press, 2007.

13. F. Galán, P. W. Ferrez, F. Oliva, J. Guàrdia, and J. del R. Millán. Feature extraction for multiclass BCI using canonical variates analysis. In IEEE International Symposium on Intelligent Singnal Processing, Alcalá de Henares, 2007.

14. F. Galán, M. Nuttin, E. Lew, P. W. Ferrez, G. Vanacker, J. Philips, H. V. Brussel, and J. del. R. Millán. An asynchronous and non-invasive brain-actuated wheelchair. In In 13th International Symposium on Robotics Research, Hiroshima, Japan., 2007.

15. Xiaorong Gao, Dingfeng Xu, Ming Cheng, and Shangkai Gao. A BCI-based environmental controller for the motion-disabled. IEEE Trans Neural Syst Rehabil Eng, 11(2):137-140, Jun 2003.

16. A. Hauser, P. E. Sottas, and J. d. R. Millán. Temporal processing of brain activity foe the recognition of EEG patterns. In Proceedings 12th Int. Conf. on Artificial Neural Nettworks, pages 1125-1130, 2002.

17. T. Hinterberger, N. Neumann, M. Pham, A. Kübler, A. Grether, N. Hofmayer, B. Wilhelm, H. Flor, and N. Birbaumer. A multimodal brain-based feedback and communication system. Exp Brain Res, 154(4):521-526, Feb 2004.

18. T. Hinterberger, S. Schmidt, N. Neumann, J. Mellinger, B. Blankertz, G. Curio, and N. Birbaumer. Brain-computer communication and slow cortical potentials. IEEE Trans Biomed Eng, 51(6):1011-1018, Jun 2004.

19. P. R. Kennedy and R. A. Bakay. Restoration of neural output from a paralysed patient by a direct brain connection. Neuroreport, 9:1707-1711, 1998. 
20. R. Krepki, B. Blankert, G. Curio, and K. R. Müller. The Berlin brain-computer interface (BBCI) towards a new communication channel for online control in gaming applications. Multimedia Tools and Applications, pages 73-90, February 2007.

21. A. Kübler and K. R. Müller. An introduction to brain computer interfacing in Towards Brain-Computer Interfacing. MIT Press, 2007.

22. M. A. Lebedev and M. A. Nicolelis. Brain-machine interfaces: past, present and future. Trends Neurosci, 29:536-546, 2006.

23. F. Lotte, M. Congedo, A. Lcuyer, F. Lamarche, and B. Arnaldi. A review of classification algorithms for eeg-based brain-computer interfaces. J Neural Eng, 4(2):R1-R13, Jun 2007.

24. M. Middendorf, G. McMillan, G. Calhoun, and K. S. Jones. Brain-computer interfaces based on the steady-state visual-evoked response. IEEE Trans Rehabil Eng, 8(2):211-214, Jun 2000.

25. J. del R. Millán. Brain-computer interfaces. In Michael A. Arbib, editor, The Handbook of Brain Theory and Neural Networks: The Second Edition. The MIT Press, 2002.

26. J. del R. Millán, P. W. Ferrez, and A. Buttfield. Non-invasive brain-machine interfaces. ESA report on studies on feasibility of non-invasive brain-machine interfaces (www.esa.int/gsp/ACT/bio/BMI.htm), 2006.

27. J. del. R. Millán and J. Mouriño. Asynchronous BCI and local neural classifiers: An overview of the Adaptive Brain Interface project. IEEE Trans. on Neural Systems and Rehabilitation Engineering, Special Issue on Brain-Computer Interface Technology, 11(2):159-161, 2003.

28. J. del. R. Millán, J. Mouriño, M. Franzé, F. Cincotti, M. Varsta, J. Heikkonen, and F. Babiloni. A local neural classifier for the recognition of EEG patterns associated to mental tasks. IEEE Trans Neural Networks, 13(3):678-686, 2002.

29. J. del. R. Millán, F. Renkens, J. Mouriño, and W. Gerstner. Non-invasive brainactuated control of a mobile robot by human EEG. IEEE Trans Biomed Eng, 51(6):1026-1033, Jun 2004.

30. J. del. R. Millán, F. Renkens, J. Mourino, and G. Wulfram. Brain-actuated interaction. Artif. Intell., 159(1-2):241-259, 2004.

31. B. Obermaier, G. R. Müller, and G. Pfurtscheller. "Virtual keyboard" controlled by spontaneous EEG activity. IEEE Trans Neural Syst Rehabil Eng, 11(4):422-426, Dec 2003.

32. S. E. Petersen, P. T. Fox, M. I. Posner, M. Mintun, and M. E. Raichle. Positron emission tomographic studies of the cortical anatomy of single-word processing. Nature, 331(6157):585-589, Feb 1988.

33. G. Pfurtscheller and C. Neuper. Motor imagery activates primary sensorimotor area in humans. Neurosci Lett, 239(2-3):65-68, Dec 1997.

34. G. Pfurtscheller and C. Neuper. Motor imagery and direct brain-computer communication. In IEEE, volume 89, pages 1123-1134, 2001.

35. J. Philips, J. del. R. Millán, G. Vanacker, E. Lew, and F. Galán. Adaptive shared control of a brain-actuated simulated wheelchair. In IEEE 10th International Conference on Rehabilitation Robotics, 2007.

36. B. Rebsamen, E. Burdet, Teo C.L., Q. Zeng, C. Guan, M. Ang, and C. Laugier. A brain control wheelchair with a P300 based bci and a path following controller. In Proc. 1st IEEE/RAS-EMBS Int. Conf. Biomedical Robotics and Biomechatronics., 2006.

37. S. J. Roberts and W. D. Penny. Real-time brain-computer interfacing: a preliminary study using bayesian learning. Med Biol Eng Comput, 38(1):56-61, Jan 2000. 
38. R. Rosen. Anticipatory systems. Pergamon press, 1985.

39. Albrecht Schmidt. Interactive Context-Aware Systems Interacting with Ambient Intelligence. IOS Press, 2006.

40. E. E. Sutter. The brain response interface: communication through visuallyinduced electrical brain responses. J. Microcomput. Appl., 15:31-45, 1992.

41. G. Vanacker, J. del. R. Millán, E. Lew, P. Ferrez, F. Galán, J. Philips, H. V. Brussel, and M. Nuttin. Context-based filtering for assisted brain-actuated wheelchair driving. Computational Intelligence and Neuroscience, 2007, 2007.

42. W. G. Walter, R. Cooper, V. J. Aldridge, W. C. McCallum, and A. L. Winter. Contingent negative variation: An electric sign of sensorimotor association and expectancy in the human brain. Nature, 203:380-4, Jul 251964.

43. J. R. Wolpaw. Brain-computer interfaces (BCIs) for communication and control: a mini-review. Suppl Clin Neurophysiol, 57:607-613, 2004.

44. J. R. Wolpaw, N. Birbaumer, D. J. McFarland, G. Pfurtscheller, and T. M. Vaughan. Brain-computer interfaces for communication and control. Clin Neurophysiol, 113(6):767-791, Jun 2002.

45. J. R. Wolpaw, D. L. McFarland, and T. M. Vaughan. Brain-Computer Interface Research at the Wadsworth Center. IEEE Trans. Rehab. Eng, 8:222-226, 2000.

46. A. Yoshino, M. Inoue, and A. Suzuki. A topographic electrophysiologic study of mental rotation. Brain Res Cogn Brain Res, 9(2):121-124, Mar 2000. 


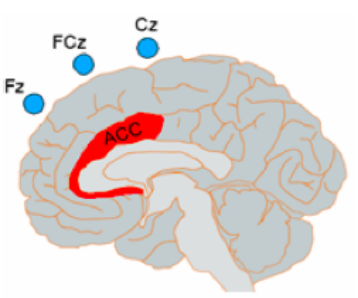

(a)

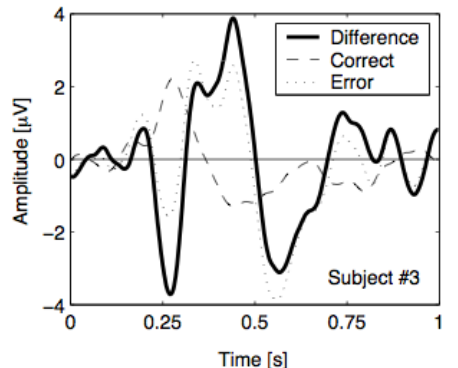

(b)

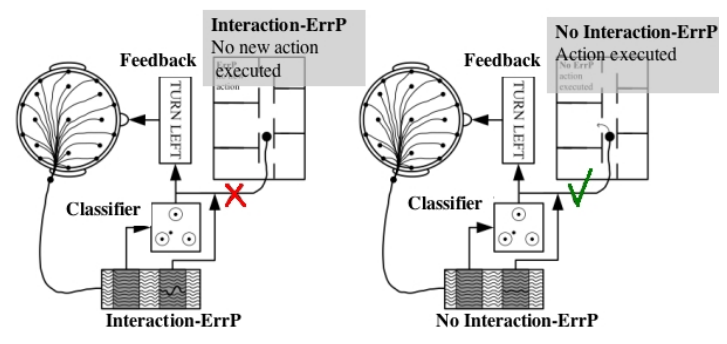

(c)

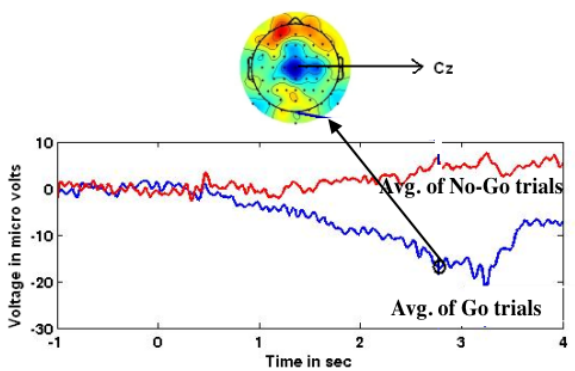

(d)

Fig. 6. (a) Position of $\mathrm{Fz}, \mathrm{FCz}$ and $\mathrm{Cz}$ electrodes with respect to Anterior Cingulate Cortex (ACC) which is the origin of ErrP (b) Average of the difference (error-minus correct) between the potentials of error and correct trials at $\mathrm{Cz}$ electrode. (c) BCI based on 'Interaction ErrP'. The BCI user receives visual feedback of indicating the output of the classification engine before the actual execution of the associated command(e.g., "turn-left"). If the feedback elicits an ErrP, this command is simply ignored and the robot will stay executing the previous command (right). Otherwise, the command is sent to the robot (left). (d) Contingent Negative Variation with a classical Go/NoGo task: external stimulus (S1) appears at time "0" secs, and imperative stimulus (S2) appears at "3.25" secs. The upper line is average of NoGo trials and lower line is average of Go trials. On the top, the scalp topography of CNV is shown at time 2.75 secs. 